\title{
Emergence of VIM-2 metallo- $\beta$-lactamase-producing Pseudomonas aeruginosa isolates in a paediatric hospital in Serbia
}

Molecular detection and surveillance of the resistance genes harboured by Pseudomonas aeruginosa are becoming increasingly important in assessing and controlling spread and colonization in hospitals, and in guiding the antibiotic treatment of infections. Metallo- $\beta$ -

lactamase (MBL)-producing $P$. aeruginosa strains are slowly but steadily increasing within hospitals, causing outbreaks and/or hyperendemic situations in some centres, mostly in the Far East and the south of Europe (Queenan \& Bush, 2007). The global dissemination of MBL-producing $P$. aeruginosa strains has also reached the Balkan region (Lepsanovic et al., 2008; Sardelic et al., 2003). The objective of our study was to detect and characterize $P$. aeruginosa isolates producing MBLs from the 400-bed paediatric tertiary care hospital Mother and Child Health Institute of Serbia 'Dr Vukan Cupic'.

During the 1-year period of sampling, 526 isolates of $P$. aeruginosa were collected from clinical specimens and routine surveillance cultures from humid hospital environments (taps, drains, incubators and ventilatory equipment) since the latter may serve as a reservoir of nosocomial pathogens, as has been recognized in numerous studies (Reuter et al., 2002; Trautmann et al., 2001).

Overall, $163(31 \%)$ of the $526 P$. aeruginosa isolates from our hospital were non-susceptible to imipenem. Of those, $148(90.8 \%)$ had lower susceptibility to meropenem as well. Isolates resistant to carbapenems were most abundant in the paediatric and surgical intensive care units $(51.5 \%)$ and in the pulmonology unit

The GenBank/EMBL/DDBJ accession numbers for the 16S rRNA gene sequences of IMD12, IMD39, IMD58, IMD85 and IMD91 are FR695882-FR695886, respectively, and those for the blaviM-2 gene sequences of IMD12, IMD39, IMD58, IMD85 and IMD91 are FR695887-FR695891, respectively.
(23.9\%, mainly from cystic fibrosis patients). The vast majority of carbapenem-resistant isolates were sampled from the respiratory tract $(76.6 \%)$ and urine $(6.1 \%)$ while isolates from other sources each represented under $4 \%$. Six isolates from the hospital environment were resistant to carbapenems; these represented $46.2 \%$ of all $P$. aeruginosa isolates collected from the hospital environment. Antibiotic susceptibility testing was carried out using the disc diffusion method, according to Clinical and Laboratory Standards Institute guidelines (CLSI, 2009). The highest susceptibility of the 163 imipenem non-susceptible isolates was to polymyxin $\mathrm{B}$ and colistin (100\%), followed by levofloxacin $(61.3 \%)$, ciprofloxacin $(49.7 \%)$, aztreonam $(42.3 \%)$, amikacin (36.2\%), ceftazidime (25.2\%), piperacillin-tazobactam (21.5\%), cefepime $(20.9 \%)$, tobramycin $(17.8 \%)$, piperacillin (17.2\%), ticarcillin-clavulanic acid $(17.2 \%)$, gentamicin $(15.3 \%)$ and ticarcillin (12.9\%). Among the tested isolates, $29.4 \%$ were pan-resistant since they were resistant to all antibiotics except polymyxins.

Out of the 163 imipenem non-susceptible isolates, $138(84.7 \%)$ had a positive combined disc test and $58(35.6 \%)$ had a positive MBL Etest. PCR assay for detection of MBL genes showed that 5/163 isolates $(3.1 \%$ of all imipenem-resistant isolates and $0.95 \%$ of all $P$. aeruginosa isolates collected during the study period), all phenotypically MBL-positive in both tests, gave amplicons with primers for the bla $a_{\mathrm{VIM}}$ gene (IMD12, IMD39, IMD58, IMD85 and IMD91) (Table 1). PCRs for $b l a_{\mathrm{IMP}}, b l a_{\mathrm{SPM}}, b l a_{\mathrm{GIM}}$ and $b l a_{\mathrm{SIM}}$ were negative. Contrary to some of the previous studies, our results revealed low specificity and positive predictive value of conventional phenotypic methods for detection of MBL-producers among imipenem non-susceptible strains. However, this phenomenon has previously been described for countries with a low prevalence of MBL-positive $P$. aeruginosa strains (Samuelsen et al., 2008).

Subsequent DNA sequencing of the bla $a_{\mathrm{VIM}}$ PCR amplicons revealed that all belonged to the $b l a_{\mathrm{VIM}-2}$ type of MBL. Nucleotide sequences of those five $b l a_{\mathrm{VIM}-2}$ genes shared, within sequenced areas, $99 \%$ identity with $b l a_{\mathrm{VIM}-2}$ genes from P. aeruginosa strains RA-02000709 and VMH-106, which are located on a class I integron. Multiple alignment of partial bla $a_{\mathrm{VIM}-2}$ nucleotide sequences from our isolates showed that they were identical within overlapping regions. Strains carrying $b l a_{\mathrm{VIM}-2}$ exhibited high-level imipenem resistance as determined by Etest (MIC >32 $\mathrm{mg} \mathrm{l}^{-1}$ ). Antibiotic susceptibility testing for VIM-2-producing isolates revealed that, apart from the polymyxins ( $100 \%$ susceptibility), levofloxacin and ciprofloxacin (80\% susceptibility) were the most active antimicrobial agents, while these strains were uniformly resistant to aminoglycosides. Interestingly, three of five VIM-2-producing isolates showed in vitro susceptibility to piperacillin, piperacillin-tazobactam and/or antipseudomonal cephalosporins, once again demonstrating the high phenotypic diversity observed in MBL-producers (Cornaglia et al., 2007). In addition, four of the VIM-2-positive strains were susceptible to fluoroquinolones, which is not very common in MBL-producing isolates (Pitout et al., 2005, 2008). Furthermore, one of the carbapenem nonsusceptible isolates collected from the hospital environment was VIM-2-positive, and it could be assumed that these serve as a possible source of nosocomial infections and further dissemination, as already stated (Trautmann et al., 2001). VIM-2producing isolates belonged to three different genotype groups as determined 
Table 1. Relevant characteristics of the five VIM-2 MBL-producing $P$. aeruginosa isolates

\begin{tabular}{|lcllcl|}
\hline Isolate & Date (day/month/year) & \multicolumn{1}{c}{ Hospital unit } & \multicolumn{1}{c|}{ Source } & PFGE profile & bla gene \\
\hline IMD12 & $25 / 11 / 2007$ & Neonatology ward & Water tap swab & I & bla $a_{\mathrm{VIM}-2}$ \\
IMD39 & $23 / 1 / 2008$ & Paediatric intensive care unit & Endotracheal aspirate & II & $b l a_{\mathrm{VIM}-2}$ \\
IMD58 & $19 / 3 / 2008$ & Surgical intensive care unit & Intra-abdominal swab & III \\
IMD85 & $20 / 5 / 2008$ & Paediatric intensive care unit & Endotracheal tube swab & III & $b l a_{\mathrm{VIM}-2}$ \\
IMD91 & $26 / 5 / 2008$ & Paediatric intensive care unit & Endotracheal aspirate & III & $b l a_{\mathrm{VIM}-2}$ \\
\hline
\end{tabular}

by ERIC2 rep-PCR and PFGE. Bearing in mind that we identified three clonal groups (PFGE) among five VIM-2producing isolates, and that $b l a_{\mathrm{VIM}-2}$ genes were identical in these isolates, it could be inferred that the prevalence of $b l a_{\mathrm{VIM}-2}$ is a consequence of horizontal gene transfer rather than clonal dissemination.

The study demonstrated a low prevalence of MBL-producing $P$. aeruginosa in our paediatric hospital. VIM-2 was the only MBL type detected, which is also the most widespread VIM-type MBL worldwide and probably the most common acquired MBL in the south of Europe.

\section{Acknowledgements}

Ministry of Science and Technological Development of Republic of Serbia, grants No. 143036 and 145069, funded this work.

\section{Branko Jovcic, ${ }^{1}$ Zorica Vasiljevic, ${ }^{2}$ Slobodanka Djukic, ${ }^{3}$ Ljubisa Topisirovic $^{1}$ and Milan Kojic ${ }^{1}$ \\ ${ }^{1}$ Institute of Molecular Genetics and Genetic Engineering, University of Belgrade, 11010 Belgrade, Serbia \\ ${ }^{2}$ Mother and Child Health Institute of}

Serbia 'Dr Vukan Cupic', 11000 Belgrade, Serbia

${ }^{3}$ Institute of Microbiology and Immunology, School of Medicine, University of Belgrade, 11010 Belgrade, Serbia

Correspondence: Branko Jovcic (lab6@eunet.rs)

CLSI (2009). Performance Standards for Antimicrobial Susceptibility Testing, 19th Informational Supplement. M100-S19. Wayne, PA: Clinical and Laboratory Standards Institute.

Cornaglia, G., Akova, M., Amicosante, G., Cantón, R., Cauda, R., Docquier, J. D., Edelstein, M., Frère, J. M., Fuzi, M. \& Galleni, M. (2007). Metallo-beta-lactamases as emerging resistance determinants in Gram-negative pathogens: open issues. Int J Antimicrob Agents 29, 380-388.

Lepsanovic, Z., Libisch, B., Tomanovic, B., Nonkovici, Z., Balogh, B. \& Füzi, M. (2008). Characterisation of the first VIM metallo- $\beta$ lactamase-producing Pseudomonas aeruginosa clinical isolate in Serbia. Acta Microbiol Immunol Hung 55, 447-454.

Pitout, J. D. D., Gregson, D. B., Poirel, L., McClure, J.-A., Le, P. \& Church, D. L. (2005). Detection of Pseudomonas aeruginosa producing metallo- $\beta$-lactamases in a large centralized laboratory. J Clin Microbiol 43, 3129-3135.
Pitout, J. D. D., Revathi, G., Chow, B. L., Kabera, B., Kariuki, S., Nordmann, P. \& Poirel, L. (2008). Metallo- $\beta$-lactamase-producing Pseudomonas aeruginosa isolated from a large tertiary centre in Kenya. Clin Microbiol Infect 14, 755-759.

Queenan, A. M. \& Bush, K. (2007).

Carbapenemases: the versatile $\beta$-lactamases. Clin Microbiol Rev 20, 440-458.

Reuter, S., Sigge, A., Wiedeck, H. \& Trautmann, M. (2002). Analysis of transmission pathways of Pseudomonas aeruginosa between patients and tap water outlets. Crit Care Med 30, 2222-2228.

Samuelsen, O., Buarø, L., Giske, C. G., Simonsen, G. S., Aasnaes, B. \& Sundsfjord, A. (2008). Evaluation of phenotypic tests for the detection of metallo- $\beta$-lactamase-producing Pseudomonas aeruginosa in a low prevalence country. J Antimicrob Chemother 61, 827830.

Sardelic, S., Pallecchi, L., Punda-Polic, V. \& Rossolini, G. M. (2003). Carbapenem-resistant Pseudomonas aeruginosa-carrying VIM-2 metallo- $\beta$-lactamase determinants, Croatia. Emerg Infect Dis 9, 1022-1023.

Trautmann, M., Michalsky, T., Wiedeck, H., Radosavljevic, V. \& Ruhnke, M. (2001). Tap water colonization with Pseudomonas aeruginosa in a surgical intensive care unit (ICU) and relation to Pseudomonas infections of ICU patients. Infect Control Hosp Epidemiol 22, 49-52. 